\title{
Class Imbalance Techniques for High Energy Physics
}

\author{
Christopher W. Murphy ${ }^{1^{*}}$ \\ 1 Insight Data Science, San Francisco, CA 94107, USA \\ * chrismurphybnl@gmail.com
}

July 27, 2019

\begin{abstract}
A common problem in a high energy physics experiment is extracting a signal from a much larger background. Posed as a classification task, there is said to be an imbalance in the number of samples belonging to the signal class versus the number of samples from the background class. In this work we provide a brief overview of class imbalance techniques in a high energy physics setting. Two case studies are presented: (1) the measurement of the longitudinal polarization fraction in same-sign $W W$ scattering, and (2) the decay of the Higgs boson to charm-quark pairs.
\end{abstract}

\section{Contents}

\begin{tabular}{lll}
\hline 1 & Overview & 2
\end{tabular}

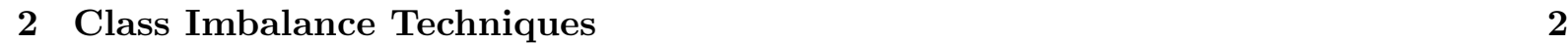

3 Longitudinal Polarization Fraction in Same-Sign $W W$ Production 4

3.1 Introduction 4

3.2 Data

3.3 Models and Training 5

3.4 Results 5

4 Higgs Boson Decays to Charm-Quark Pairs 8

4.1 Introduction 8

$\begin{array}{lll}4.2 & \text { Data } & 8\end{array}$

$\begin{array}{llr}4.3 & \text { Models and Training } & 9\end{array}$

\begin{tabular}{lll}
\hline 4.4 & Results & 10
\end{tabular}

$\begin{array}{lll}5 \text { Discussion } & 10\end{array}$

\begin{tabular}{ll}
\hline A Glossary & 11
\end{tabular}

References 11 


\section{Overview}

The Large Hadron Collider (LHC) has been an incredibly successful experiment. To date it has discovered the Higgs boson, and measured hundreds, if not thousands, of other processes to be consistent with the predictions of the Standard Model (SM) 11. A common problem in making these measurements is extracting a signal from a much larger background. Occasionally in this situation there is a single feature that is powerful enough to discriminate the signal from the large background. An example of this the Higgs boson decaying to two photons where the invariant mass of the photon pair is the discriminating observable [2, 3]. More often however a multi-variate analysis of many features needs to be performed. Machine learning (ML) and deep learning (DL) are well suited for such tasks. Therefore it is not surprising that ML and DL have become, and will likely continue to be, an important part of the success of the LHC program. See Refs. [4, 5, 6, 7, for some recent reviews.

If one treats the extraction of a signal from a much larger background as a classification problem there is an imbalance in the number of sample belonging to the signal class versus the number of events from the background class. In the machine learning community techniques for learning from imbalanced data are well established. There is now even a software package, imbalanced - learn [8], dedicated to this task. In high energy physics there do not appear to be many cases where imbalanced learning techniques were explicitly used. However the measurement of the time-integrated $C P$ asymmetry in $D^{0} \rightarrow K_{S}^{0} K_{S}^{0}$ decays by $\mathrm{LHCb}[9$ is one such example. In particular LHCb classified the $D^{0}$ decay signal from its background using the analysis methods developed in Refs. [10, 11]. An alternative approach to classification with imbalance techniques is using an anomaly detection framework. There are several examples of this in high energy physics [12, 13, 14, 15].

Given the lack of examples where imbalanced learning techniques were used in high energy physics the purpose of this note is two-fold. Firstly, in Section 2, we aim to provide a brief overview of modern class imbalance techniques in a high energy physics setting, introducing novel loss functions and a data resampling technique. Secondly, we provide two case studies of how class imbalance techniques can be used in high energy physics settings. The first case, presented in Sec. 3, is the measurement of the longitudinal polarization fraction in same-sign $W W$ scattering. We find a significant improvement in the performance of the machine learning models used in the longitudinal $W W$ study, while no significant improvement in performance is found in the deep learning models tested. The second study is the decay of the Higgs boson to charm-quark pairs, which follows in Sec. 4. Our Higgs-to-charm tagger gives a 14\% improvement in the background rejection rate. Another application of these techniques is training directly on experimental data [16, 17, 18. Conclusions are then given in Sec. 5. Much of the code for this project is available at [19].

\section{Class Imbalance Techniques}

There is no definitive answer to the question: What should one do when dealing with imbalanced data? The answer will depend on the data in question, see [20] for a study of benchmark datasets. In this Section we present a few approaches one might try to improve performance on an unbalanced dataset.

Using the accuracy of a classifier as a metric can be misleading. (See Table. 3 for a glossary of model evaluation terms used in this work.) Consider a model that predicts that every sample 
to be background. The accuracy of this model is $A=1 /(1+r)$, where $r$ is the ratio of the number of signal events to the number of background events. Although this model would be highly accurate if the data were sufficiently imbalanced, it would not be useful as it says nothing about the signal, which is what we were interested in to begin with. For this reason accuracy is not a recommended metric in this setting. The ROC curve is a good general purpose metric, providing information about the true and false positive rates across a range of thresholds, and the area under the ROC curve $(A U C)$ is a good general purpose, single number metric. The $A U C$ score focuses on true negatives, which are less informative than false positives and false negatives when the data is imbalanced. Therefore we recommend the precision-recall curve as the metric to use on imbalanced data, or the average precision score if a single number is preferred.

One might also try to balance the training set either by under-sampling [21, 22, 23, 24, 25] the majority class, oversampling the minority class [26, 27, or a combination of over- and undersampling [28, 29]. Oversampling runs the risk of overfitting, and training with oversampling takes longer because of the additional data. For these reasons we will focus on under-sampling in this work. In particular, we will use random under-sampling to create a balanced random forest [30, 31]. Analogous procedures exist for creating a balanced boosted decision trees [32] and making balanced batches to feed into a neural network. The algorithm for how the balanced random forest makes classifications is as follows: (1) take bootstrap samples from the original dataset, (2) balance each sample by downsampling randomly, (3) learn a decision tree from each sample, (4) make predictions based on a majority vote. It is the second step of this process that is absent in a standard random forest. Even if this does not lead to a gain in performance training is faster with this approach because less data is used.

Lastly, one might consider making changes to the algorithms being used [33, 34, 35]. A simple example of this is if a metric such as precision, recall, or $F_{1}$ score is being used, its decision threshold can be optimized to maximize performance. Another approach is to adjust the class weights in the loss function, creating a larger penalty for misclassifying an example from the minority class. Consider the cross entropy loss function used for binary classification!

$$
L=-y \log (p)-(1-y) \log (1-p),
$$

where $y$ is the ground-truth class with $y=1$ for the signal class, and $p$ is the model's estimated probability that a given event belong to the signal class. Weighting the loss function can be implemented as follows

$$
L=-\alpha y \log (p)-(1-\alpha)(1-y) \log (1-p),
$$

where the class weight hyperparameter, $\alpha$, takes values between 0 and 1 in this notation. Ref. [36] took this one step further, introducing the focal loss function

$$
L=-\alpha y(1-p)^{\gamma} \log (p)-(1-\alpha)(1-y) p^{\gamma} \log (1-p),
$$

where the focusing parameter, $\gamma>0$, puts the focus on hard, misclassified examples. In doing so it down weights the easy-to-classify negatives that can overwhelm a model during training in the presence of class imbalance. Focal Loss is an optimal classifier just as cross entropy or mean square error are. One way to see this is Focal Loss produces a concave ROC curve (given sufficiently large statistics), which is equivalent to being optimized by the likelihood ratio [37].

\footnotetext{
${ }^{1}$ The loss function for a single sample is shown to reduce clutter. For tree based models, such as random forest, our baseline loss function is the CART loss function with Gini impurity not the cross entropy. However the logic of modifying the baseline loss function is the same.
} 


\section{Longitudinal Polarization Fraction in Same-Sign $W W$ Produc- tion}

\subsection{Introduction}

Same-sign $W W$ production at the LHC is the vector boson scattering (VBS) process with the largest ratio of electroweak-to-QCD production. As such it provides a great opportunity to study whether the discovered Higgs boson leads to unitary longitudinal VBS, and to search for physics beyond the SM (BSM) [38, 39]. The ATLAS and CMS experiments have observed electroweak same-sign $W W$ production in the two jet, two same-sign lepton final state in $13 \mathrm{TeV}$ $p p$ collisions with significances of $6.9 \sigma$ [40] and $5.5 \sigma$ [41], respectively. Confirming or refuting the unitarity of VBS requires not just a measurement of $p p \rightarrow j j W^{ \pm} W^{ \pm}$, but of the fraction of these events where both $W$ s are longitudinally polarized (LL fraction).

Prospects for the extraction of the longitudinal component of $W^{ \pm} W^{ \pm}$scattering during the High-Luminosity phase of the LHC (HL-LHC) were studied in Refs. [2, 43, 44]. The fraction of longitudinally polarized events is predicted to be only $r \approx 0.07$ in the SM at large dijet invariant mass $\left(m_{j j}\right)$ [43] making this a challenging measurement. Using the difference in the azimuthal angle of the two jets $\left(\Delta \phi_{j j}\right)$ as a discriminant, the significance for the observation of the LL fraction is expected to be up to $2.7 \sigma$ with $3000 \mathrm{fb}^{-1}$ of integrated luminosity [43].

The observation significance can be improved through the use of deep learning [45, 46]. Ref. [45] regressed on the angles between the charged leptons in their parent boson's rest frame and the $W$ boson's direction of motion, whereas Ref. [46] treated this as a binary classification problem distinguishing between events where both $W$ s were longitudinally polarized versus when one or none of the $W$ s were polarized. In the classification setting it is important to keep in mind that the predicted LL fraction is small, and thus there is an imbalance in the number of events belonging to the class $N\left(W_{L}\right)=2$ versus the class $N\left(W_{L}\right)<2$ (LL class vs. TT+LT class). We proceed treating this as a classification problem with imbalanced classes.

\subsection{Data}

Events for the process $p p \rightarrow j j W^{ \pm} W^{ \pm}$are simulated with MadGraph5aMC@NLO [47]. 1.6 $10^{5}$ events are generated with $m_{j j}>150 \mathrm{GeV}$ and all other parameters fixed to their default values in MadGraph, i.e. $\sqrt{s}=13 \mathrm{TeV}$. The $W$ s are left undecayed; that is to say the four-vectors of the $W$ s are used as features rather than the four-vectors of the charged leptons and MET. We content ourselves with training on less realistic simulated data as we are interested in seeing a change in performance relative to a baseline, and we will show that our baseline models perform comparably well to the models in Ref. [46]. We also study what happens when the cuts match those of the prospective HL-LHC studies [42, 43]. The number of simulated events passing the following cuts, $m_{j j}>500 \mathrm{GeV}$ and $\Delta \eta_{j j}>2.5$, is approximately $2.5 \cdot 10^{4}$. We find these cuts do not qualitatively affect our conclusions, and present our results from the larger simulated dataset with $m_{j j}>150 \mathrm{GeV}$.

The four-vectors $\left(E_{i}, p_{T, i}, \eta_{i}, \phi_{i}\right)$ of the two jets and two $W \mathrm{~s}$ are used as features, $i=$ $\left\{j_{1}, j_{2}, W_{1}, W_{2}\right\}$. Note that in this case study the "jets" are partons from the hard scattering process and are not showered or hadronized. The subscripts 1 and 2 are used to indicate the jet or $W$ with the larger or smaller transverse momentum, e.g. $p_{T, j_{1}}>p_{T, j_{2}}$. This is an important feature engineering step that improves the performance of classifiers that is not done by default in MadGraph. In addition, the following high-level features are added. From the jet system we add 
the invariant mass, the difference in pseudorapidity $\left(\Delta \eta_{j j}\right)$, and the difference in the azimuthal angle. We also add the four-vector $\left(E_{W W}, p_{T, W W}, \eta_{W W}, \phi_{W W}\right)$ and invariant mass $\left(m_{W W}\right)$ of the $W W$ system, bringing the total number of features to 24 . Clearly we have introduced some redundancy into our set of features. This is done to increase the rate of learning of our models.

\subsection{Models and Training}

In addition to using $\Delta \phi_{j j}$ as a discriminating observable, we use the following models. For classical machine learning we use a random forest (RF) as a baseline, and look to use a change in performance from weighting or balancing. We use the imbalanced - learn [8] implementation of balanced random forest, and use scikit - learn [48] for the other random forests. A grid search using a subset of the data is performed to optimize the hyperparameters of the random forests. The top performing forests have no maximum depth. The deep learning models are fully-connected neural networks (DNNs) implemented in Keras [49] with a TensorFlow [50] backend. Our baseline DNN has a cross entropy loss function. The variants we are looking to test are a DNN with a focal loss function, and a DNN with a cross entropy loss but where the training batches have been balanced. All of our neural networks have 2 hidden layers each with 150 neurons, He initialization, and ReLU activation functions. Batch normalization is performed to speed up the learning process, dropout is applied at a $50 \%$ rate for regularization, and the Adam algorithm is used to optimize the parameters of the DNN.

A five-fold cross validation is performed for each for model. The folds are stratified based on the size of the class imbalance. The classical ML models are trained on a MacBook Pro, while the DL models are trained on a GPU hosted by Amazon Web Services (p2.xlarge). For the DNNs, a batch size of 1024 is used in training. We find this is the largest batch size we can use with this dataset without suffering a loss in performance 2 Training for DNNs runs until there is no decrease in the loss function for 10 consecutive epochs. An upper limit of 300 epochs is imposed, but in practice this is never reached.

\subsection{Results}

Table 1 shows the results of the cross validation with performance being reported as the mean \pm the standard deviation of the five folds. Recall the ML models are trained on a CPU, and DL models are trained on a GPU, accounting for the typically longer training time of the ML models. As advertised, the models with balanced training data train faster than their unbalanced counterparts. In particular there is a reduction in training time by a factor of 2-3. The weighted random forest has a $1 \%$ higher average precision than the baseline random forest. However this is the same size as the standard deviation of the average precision of each model. On the other hand, the $A U C$ of the balanced random forest shows a $1.5 \%$ improvement with respect to the baseline RF. This difference is significant given the size of the uncertainties. For the DNN with focal loss there is perhaps a marginal improvement in performance with respect to the baseline DNN, but this difference is not significant. The balanced batch DNN performs worse than the baseline DNN likely due to the smaller amount of the training data it uses.

The significance that the LL fraction is non-zero is estimated as follows. We assume $3000 \mathrm{fb}^{-1}$ of data is collected and that the $W$ s decay to electrons or muons. The discriminant functions for the various models are shown in Figure 1. These are histograms for a model's estimated

\footnotetext{
${ }^{2}$ With a batch size of 1024 one epoch took about one second on the GPU versus three seconds on the CPU. Conversely with a batch size of 50 one epoch took about 30 seconds on the GPU versus 15 seconds on the CPU.
} 


\begin{tabular}{|c||c|c|c|c|}
\hline Model & Time/Fold $[\mathrm{s}]$ & Average Precision & $A U C$ & Significance $[\sigma]$ \\
\hline \hline$\Delta \phi_{j j}$ & $0.2 \pm 0.0$ & $0.081 \pm 0.003$ & $0.647 \pm 0.007$ & $2.4 \pm 0.1$ \\
\hline Random Forest & $334 \pm 9$ & $0.143 \pm 0.010$ & $0.758 \pm 0.007$ & $4.8 \pm 0.2$ \\
Weighted RF & $287.3 \pm 1.5$ & $0.153 \pm 0.011$ & $0.767 \pm 0.009$ & $5.0 \pm 0.3$ \\
Balanced RF & $92.7 \pm 0.8$ & $0.149 \pm 0.009$ & $0.773 \pm 0.007$ & $4.6 \pm 0.4$ \\
\hline Deep Neural Network & $219 \pm 24$ & $0.230 \pm 0.015$ & $0.820 \pm 0.007$ & $6.5 \pm 0.3$ \\
DNN w/ Focal Loss & $229 \pm 38$ & $0.232 \pm 0.020$ & $0.821 \pm 0.011$ & $6.3 \pm 0.4$ \\
Balanced Batch DNN & $89 \pm 17$ & $0.182 \pm 0.013$ & $0.797 \pm 0.008$ & $5.3 \pm 0.3$ \\
\hline
\end{tabular}

Table 1: Results of the five-fold cross validation for classifying LL events from TT+LT events in $p p \rightarrow j j W^{ \pm} W^{ \pm}$. The models with balanced training data train faster than their unbalanced counterparts, reducing training time by a factor of 2-3. The $A U C$ of the balanced random forest shows a $1.5 \%$ improvement with respect to the baseline RF. This difference is significant given the size of the uncertainties. See the text more for details.

probability that a given event belongs to the signal class. If this probability is larger than the decision threshold then that event is predicted to be signal. For metrics that makes predictions, as opposed to just predicting probabilities, the decision threshold can be optimized to extremize the score of the metric. The red histograms are for events that are ground truth signal, $f_{\mathrm{LL}}$. Similarly, the blue histograms are for events that are ground truth background, $f_{\mathrm{TT}+\mathrm{LT}}$. The top row shows the random forest models, and the bottom row shows the deep neural network models. We fit to the discriminant distributions in Fig. 1, and extract the most likely value of the LL fraction and its uncertainty using the method of maximum likelihood. The log-likelihood function is

$$
-\log (\mathcal{L}(\mu))=-\sum_{e} \log \left(\mu f_{\mathrm{LL}}\left(p_{e}\right)+(1-\mu) f_{\mathrm{TT}+\mathrm{LT}}\left(p_{e}\right)\right)
$$

where $\mu$ is the LL fraction, and $f_{i}$ is the pdf for the probability that an ML model predicts an event to be class $i$. The various models have different levels of confidence in their predictions, which accounts for the imperfect correlations between a model's $A P$ and $A U C$ scores and its significance for a non-zero LL fraction.

Our neural network models and $\Delta \phi_{j j}$ observable outperform the analogous models in Ref. [46]. We find this is primarily due to our less restrictive cut on $m_{j j}$. The $A U C$ of our models monotonically decreases as the cut on $m_{j j}$ increases reaching values comparable to those found in Ref. 46] around $m_{j j} \sim 800 \mathrm{GeV}$. Having identified the $m_{j j}$ cut as the main difference in performance, it would appear that hadronization effects do not play an important role in the performance of these models. We note that our neural networks achieves comparable performance to that of Ref. [46] despite having only two hidden layers instead of 10, which is not surprising given the relative simplicity of this data. The advantage of having fewer hidden layers is a reduction in training time. On the other hand, our random forest models underperform compared to the analogous boosted decision tree of Ref. [46], possible due to being trained on less data or perhaps simply due to inherent differences between the models. Nevertheless our goal was determine the change in performance relative to a baseline, which we were able to accomplish. 

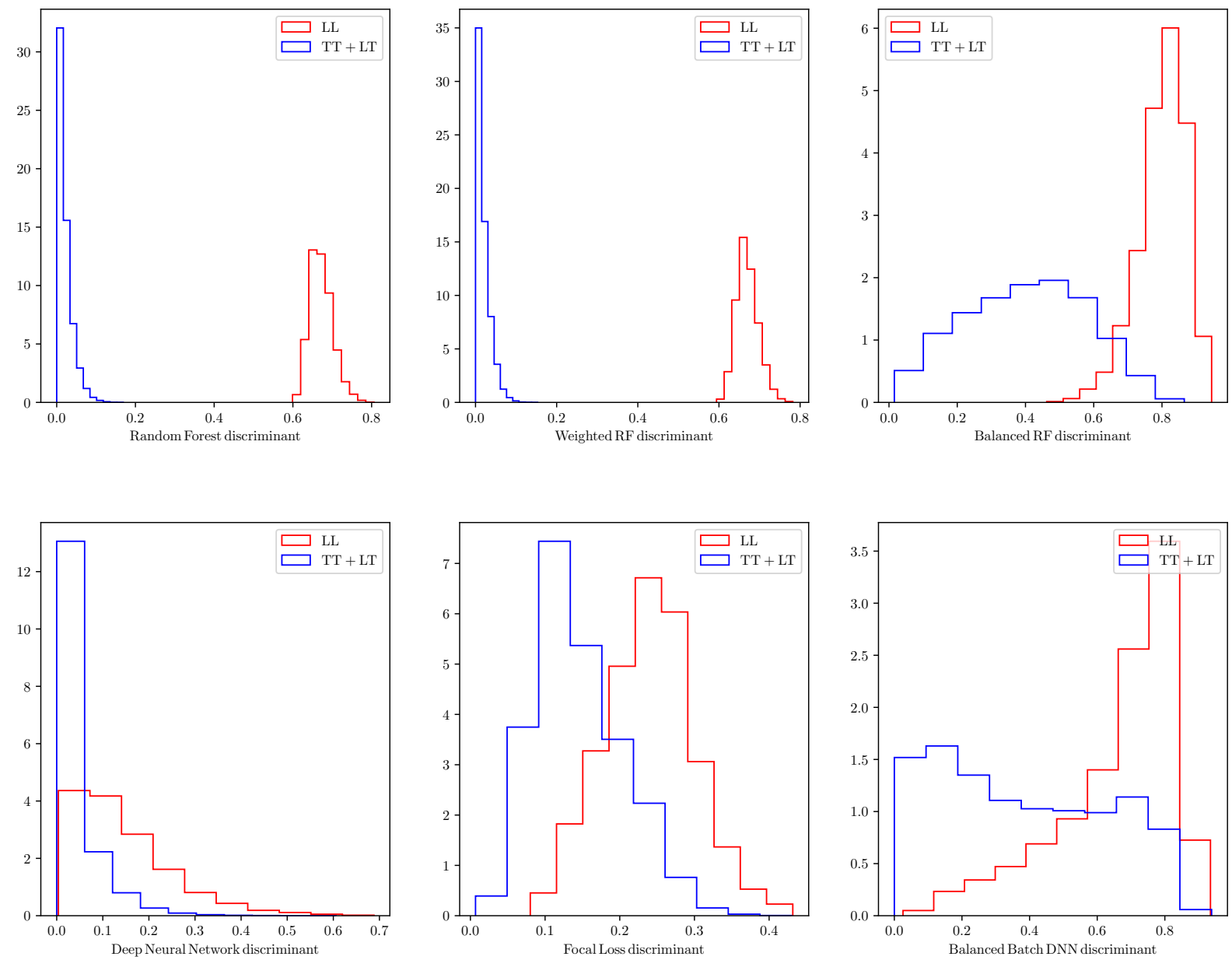

Figure 1: Histograms for the probability the event will be predicted to be an LL event when it is actually an LL event (red distributions) or when it is actually an TT+LT event (blue distributions). The top row shows the random forest models, and the bottom row shows the DNN models. The various models have different levels of confidence in their predictions, which accounts for the imperfect correlations between a model's $A P$ and $A U C$ scores and its significance for a non-zero LL fraction. 


\section{Higgs Boson Decays to Charm-Quark Pairs}

\subsection{Introduction}

The second application of class imbalance techniques we explore in this note is to the measurement of Higgs boson decay to charm-quark pairs. Searches for the decay of the Higgs boson to charm-quarks have produced only weak limits to date. ATLAS reported an upper limit of 110 times the SM rate for the process $p p \rightarrow Z h \rightarrow \ell^{-} \ell^{+} c \bar{c}$ [51]. LHCb instead considered the associated production of both $W \mathrm{~s}$ and $Z \mathrm{~s}$ in range $2<\eta<5$, and set a limit of 6,400 times the SM rate [52]. A result of these weak limits is that direct limits on the charm Yukawa coupling are correspondingly weak. Stronger bounds can be obtained indirectly, e.g. through global fits [53], among other methods. However there are assumptions build into any indirect analysis. The limit on the charm Yukawa coupling at HL-LHC is projected to get down to about 2.2 times the SM rate [54] (see also [55]). Based on this projection an observation of $h \rightarrow c \bar{c}$ is not expected at HL-LHC motivating ways to improve the analysis, although this projected limit should still be useful in constraining certain BSM physics.

One reason for the weak limits on $h \rightarrow c \bar{c}$ is in the SM the rate for $h \rightarrow b \bar{b}$ is about 20 times larger $(r \approx 0.05)$ than the rate for $h \rightarrow c \bar{c}[56]$. In contrast with $h \rightarrow c \bar{c}$, the decay of the Higgs boson to bottom-quarks has been observed by both ATLAS [57] and CMS [58] The analyses of Refs. [51, 52, 57, 58] rely on tagging the flavor of the jets, which involves discriminating charm initiated jets from bottom jets, or vice versa, and discriminating heavy from light flavored jets ${ }^{3}$ The use of flavor tagging explicitly links the measurements of $h \rightarrow b \bar{b}$ and $h \rightarrow c \bar{c}$ [60, 61].

To perform the flavor tagging LHCb used their standard, state-of-the-art heavy flavor tagger [62], while ATLAS trained boosted decision trees to separate charm from light jets and charm from bottom jets with a procedure analogous to how they train their standard bottom tagger [63, 64]. The use of general purpose flavor tagging algorithms is less than ideal for the specific task of identifying Higgs decays to charms. This was recognized in Ref. [65], which made a dedicated double-charm tagger for $h \rightarrow c \bar{c}$. We also advocate making a dedicated $h \rightarrow c \bar{c}$ tagger for the following reason. The standard heavy flavor tagging algorithms are not optimized for the imbalance in the expected number of $h \rightarrow c \bar{c}$ versus $h \rightarrow b \bar{b}$ events. For example, QCD produces roughly equal numbers of bottoms and charms at invariant masses relevant for Higgs physics. Given the statistical nature of heavy flavor tagging, an imbalance in the number of $b \bar{b}$ and $c \bar{c}$ decays will lead to worse performance in identifying the Higgs to charm events. As such this is a well motivated arena for applying class imbalance techniques. Here we are assuming an SM-like rate for $h \rightarrow c \bar{c}$. If some BSM physics makes the experimental rate for $h \rightarrow c \bar{c}$ much larger than expected this would invalidate our argument (which would be a small price to pay for the discovery of the breakdown of the SM). The rest of this case study delivers proof of principle that it is possible to improve tagging efficiency of $h \rightarrow c \bar{c}$ events through the use of the class imbalance techniques.

\subsection{Data}

We consider associated Higgs production at an $e^{+} e^{-}$collider as an observation of $h \rightarrow c \bar{c}$ is not expected at HL-LHC. Specifically, the process under consideration is $e^{+} e^{-} \rightarrow Z h \rightarrow$ $\ell^{+} \ell^{-} Q \bar{Q}$ with $\ell=e$ and $\mu$, and $Q=b$ or $c$. A total of $2 \cdot 10^{5}$ events are simulated with MadGraph5aMC@NLO [47] with Pythia6 [66] used for parton showering and hadronization. Half

\footnotetext{
${ }^{3} \mathrm{~A}$ complementary approach is to exclusively search for charmed-hadrons [59].
} 
the simulated events are $h \rightarrow b \bar{b}$ and the other half are $h \rightarrow c \bar{c}$. We focus on the binary classification problem of $h \rightarrow c \bar{c}$ versus $h \rightarrow b \bar{b}$ as existing tagging algorithms perform well at distinguishing heavy from light flavors, see e.g. 62. The center-of-mass energy of the collisions is $\sqrt{s}=250 \mathrm{GeV}$. Jets are clustered using the FastJet [67] implementation of the anti- $k_{t}$ clustering algorithm [68] with radius parameter $R=0.4$. We require at least two jets each with $p_{T}>10 \mathrm{GeV}$. Similarly, we require the leptons to be oppositely charged, and to each have $p_{T}>10 \mathrm{GeV}$.

The four-vector of each lepton and the two leading jets are used as features. Here we use the mass, $m$, of the jet or lepton instead of the particle's energy, which was used in the same-sign $W W$ case study. It is unlikely that the mass of the jet could be measured with enough precision in an actual experiment to distinguish a charm initiated jet from a bottom jet. However the mass of the jet is a proxy for the lifetime of the initiating particle of the jet, which is a feature flavor tagging algorithms exploit, see e.g. [51]. The four-vectors of the dilepton and dijet systems, which reconstruct the $Z$ and Higgs bosons, respectively, are also included in our feature set. A cut on the invariant mass of the jets is imposed, $95<m_{j j} / \mathrm{GeV}<155$, to concentrate on resonant Higgs production. All of the above cuts and requirements reduce the number of simulated events to approximately $8.9 \cdot 10^{4}$. We include $\Delta R=\sqrt{\left(\eta_{j 1}-\eta_{j 2}\right)^{2}+\left(\phi_{j 1}-\phi_{j 2}\right)^{2}}$ between the two jets as a feature as well as the rescaled mass drop observable, ISY, and the radius of the dijet system, $R_{j j}$,

$$
I S Y=\frac{\max \left(m_{j 1}, m_{j 2}\right) \Delta R}{m_{j j}}, \quad R_{j j}=\frac{m_{j j}\left(p_{T, j 1}+p_{T, j 2}\right)}{p_{T, j j} \sqrt{p_{T, j 1} p_{T, j 2}}} .
$$

Lastly, as bottom- and charm-quarks are oppositely charged, we look at the charge of the jets as defined in [69]

$$
\mathcal{Q}_{\kappa}^{j}=\frac{1}{\left(p_{T, j}\right)^{\kappa}} \sum_{p \in j} Q_{p}\left(p_{T, p}\right)^{\kappa}
$$

where the charge, $\mathcal{Q}$, of a jet, $j$ is the $p_{T}$ weighted sum of the charges, $Q$, of all the partons, $p$, in the jet. We use $\kappa=0.4$ in this work. Of course only the overall magnitude of the jet charges differ between bottom and charm Higgs decays. Therefore, in addition to the charge of each jet, we include the product of the jet charges, the absolute value of the difference of the jet charges, and the charge of the dijet system, bringing our total number of features to 30 .

\subsection{Models and Training}

Our heavy flavor tagging model is a LightGBM [70] (LGBM), which is a gradient boosted decision tree where the trees are grown in a depth first rather than breadth first fashion. The name Light comes from the fact that the training time is often greatly reduced with this construction of the trees. Our model combines a mere 50 trees in series, and each tree is allowed to have a maximum depth of 10 with all other hyperparameters fixed to their default values. We take as our baseline heavy flavor tagger a LightGBM with an unweighted loss function, and compare its performance against a LightGBM with weighting $\alpha=1-r$.

For model evaluation we again perform a stratified five-fold cross validation. We test three scenarios. In the first test we assume the rate for $h \rightarrow c \bar{c}$ in equivalent to the rate for $h \rightarrow b \bar{b}$. Here we use the baseline LGBM with unweighted loss function. In this case there is no class imbalance implying there must be some BSM physics in this scenario. We randomly select $4.0 \cdot 10^{4}$ bottom and $4.0 \cdot 10^{4}$ charm events from our full simulated dataset, and perform the 
cross validation on this sample. For the second test we again use the unweighted, baseline model, but perform the cross validation on dataset with SM-like class imbalance. In particular we randomly select $4.0 \cdot 10^{4}$ bottom and $2.0 \cdot 10^{3}$ charm events from our full simulated dataset. For the third and final test we reuse the dataset from the second test, but use our class imbalance optimized LGBM with weighting hyperparameter $\alpha=1-r \approx 0.95$.

\subsection{Results}

The results of our three $h \rightarrow c \bar{c}$ tagging tests are given in Table 2 with the rows from top to bottom corresponding to the 1st, 2nd, and 3rd scenarios described in previous subsection. For each scenario we consider two signal efficiency working points, a looser selection of $\epsilon_{h \rightarrow c \bar{c}}=$ $T P R=0.2$ and a tighter selection of $\epsilon_{h \rightarrow c \bar{c}}=0.8$. We report the background rejection rate, $\epsilon_{h \rightarrow b \bar{b}}=F P R$, for each of these working points. The inverse of the background rejection rate is largest in the scenario without class imbalance. The performance of both tagging models is worse in the presence of class imbalance. However the weighted LGBM outperforms the baseline tagging model in the presence of class imbalance, demonstrating proof of principle that class imbalance techniques can be used to improve the performance of algorithms used to identify $h \rightarrow c \bar{c}$ events. In particular, there is a $14 \%$ increase in $1 / \epsilon_{h \rightarrow b \bar{b}}$ with loose selection criteria when the class imbalance optimized model is used.

We also report the average precision, and average precision normalized by the imbalance ratio. The average precision is significantly higher in the scenario without class imbalance. However when the average precision is normalized to the imbalance ratio, which constitutes the naïve expectation for the $A P$ score, higher values are found when the data is imbalanced.

Lastly, we investigate which features are important for the classification. Using the feature importance of the LGBM the charges of the heavy flavor jets and the associated engineered features do not play a significant role in discriminating charm initiated jets from bottom jets. This is in contrast with studies of light flavored jets [71. A possible explanation for this is the heavy flavored hadrons have more possible decay chains 4 In particular, a neutral meson may oscillate or there might be a cascade decay that spoils the correlation between the charges of the partons in the jet and the charge of the particle that initiated the jet. Again using the feature importance of the LGBM, we find the the four-vectors of the leptons and the four-vector of the reconstructed $Z$ boson also do not play a major role in discriminating charm initiated jets from bottom jets.

\section{Discussion}

Extracting a signal from a much larger background is a common problem in high energy physics. Posed as a classification task, there is said to be an imbalance in the number of samples belonging to the signal class versus the number of samples from the background class. Imbalanced learning techniques are not commonly used, explicitly anyways, in high energy physics. Given this lack of use we first provided a brief overview of modern class imbalance techniques in a high energy physics setting, introducing novel loss functions and a data resampling technique. We then presented two case studies illustrating these techniques. The first study is the measurement of the longitudinal polarization fraction in same-sign $W W$ scattering. We found a significant

\footnotetext{
${ }^{4}$ This is one of the main systematic uncertainties in measuring asymmetric heavy quark hadroproduction 72 , 73, 74, 75.
} 


\begin{tabular}{|c|c||c|c|c|c|c|}
\hline Model & $\alpha$ & $r$ & $\epsilon_{h \rightarrow c \bar{c}}$ & $1 / \epsilon_{h \rightarrow b \bar{b}}$ & Average Precision & $A P / r$ \\
\hline \hline LightGBM & $\frac{1}{2}$ & 1 & $\begin{array}{c}0.2 \\
0.8\end{array}$ & $\begin{array}{c}38.8 \pm 2.6 \\
1.7 \pm 0.0\end{array}$ & $0.719 \pm 0.004$ & $0.7 \pm 0.0$ \\
\hline LightGBM & $\frac{1}{2}$ & 0.05 & $\begin{array}{c}0.2 \\
0.8\end{array}$ & $\begin{array}{c}30.9 \pm 5.2 \\
1.6 \pm 0.1\end{array}$ & $0.166 \pm 0.008$ & $3.3 \pm 0.2$ \\
\hline Weighted LGBM & $1-r$ & 0.05 & $\begin{array}{c}0.2 \\
0.8\end{array}$ & $\begin{array}{c}35.1 \pm 8.9 \\
1.5 \pm 0.1\end{array}$ & $0.161 \pm 0.011$ & $3.2 \pm 0.2$ \\
\hline
\end{tabular}

Table 2: The results of our three $h \rightarrow c \bar{c}$ tagging tests. We report the background rejection rate, $\epsilon_{h \rightarrow b \bar{b}}=F P R$, for two signal efficiency working points, $\epsilon_{h \rightarrow c \bar{c}}=T P R=0.2$ (loose), 0.8(tight). There is a $14 \%$ increase in $1 / \epsilon_{h \rightarrow b \bar{b}}$ with loose selection criteria when the class imbalance optimized model is used, 3rd versus 2 nd row, demonstrating proof of principle that class imbalance techniques can be used to improve the performance of algorithms used to identify $h \rightarrow c \bar{c}$ events. We also report the $A P$, and $A P / r$, which is given to one decimal place for better readability.

improvement in the performance of the classic ML models used in the longitudinal $W W$ study, while no significant improvement in performance was found in the deep learning models tested. With that being said our neural networks achieves comparable performance to that of Ref. 46] despite having only two hidden layers instead of 10 . Given that there are only $\mathcal{O}(10)$ features in this dataset it is not surprising that a very deep network did not continue to improve performance. Having fewer hidden layers with all else being equal results in a reduction in training time. The second case is the decay of the Higgs boson to charm-quark pairs. We delivered proof of principle that it is possible to improve tagging efficiency of $h \rightarrow c \bar{c}$ events through the use of the class imbalance techniques. In particular, our Higgs-to-charm tagger with loose selection criteria gave a $14 \%$ improvement in the background rejection rate.

Acknowledgements We thank Eder Izaguirre and Brian Shuve for collaborations on a previous incarnation of the $h \rightarrow c \bar{c}$ tagging study. We would also like to thank Emmanuel Ameisen, Sally Dawson, Samuel Homiller, and Marc-André Pleier for useful discussions.

\section{A Glossary}

See Table 3 for a glossary of model evaluation terms used in this work.

\section{References}

[1] M. Tanabashi et al., Review of particle physics, Phys. Rev. D 98, 030001 (2018), doi:10.1103/PhysRevD.98.030001.

[2] V. Khachatryan et al., Observation of the diphoton decay of the Higgs boson and measurement of its properties, Eur. Phys. J. C74(10), 3076 (2014), doi:10.1140/epjc/s10052-0143076-z, 1407.0558. 


\begin{tabular}{|c|c|c|}
\hline Metric & Symbol & Definition \\
\hline Accuracy & $A$ & $A=(T P+T N) /(F N+F P+T N+T P)$ \\
\hline Area Under the ROC Curve & $A U C$ & $A U C=\int_{0}^{1} d(T P R)[1-F P R(T P R)]$ \\
\hline Average Precision & $A P$ & $A P=\sum_{n}\left(R_{n}-R_{n-1}\right) P_{n}$ \\
\hline Decision Threshold & $n$ & $\begin{array}{l}\text { if } p>n \text { for a given event, then that event is predicted } \\
\text { to be signal }\end{array}$ \\
\hline F1 score & $F_{1}$ & $F_{1}=2 P \cdot R /(P+R)$ \\
\hline False Negative & $F N$ & a signal event that is predicted to be background \\
\hline False Positive & $F P$ & a background event that is predicted to be signal \\
\hline False Positive Rate & $F P R$ & $F P R=F P /(F P+T N)$ \\
\hline Ground Truth Class & $y$ & $\begin{array}{l}y=1 \text { if the event is truly a signal event, and } y=0 \text { if } \\
\text { it is background }\end{array}$ \\
\hline Preci & $P$ & $P=T P /(F P+T P)$ \\
\hline Probability Estimate & $p$ & $\begin{array}{l}\text { a model's estimated probability that a given event be- } \\
\text { longs to the signal class }\end{array}$ \\
\hline Recall & $R$ & $R=T P /(F N+T P)$ \\
\hline True Negative & $T N$ & a background event that is predicted to be background \\
\hline True Positive & $T P$ & a signal event that is predicted to be signal \\
\hline True Positive Rate & $T P R$ & $T P R=R$ \\
\hline
\end{tabular}

Table 3: Glossary of model evaluation terms used in this work.

[3] G. Aad et al., Measurement of Higgs boson production in the diphoton decay channel in pp collisions at center-of-mass energies of 7 and $8 \mathrm{TeV}$ with the ATLAS detector, Phys. Rev. D90(11), 112015 (2014), doi:10.1103/PhysRevD.90.112015, 1408.7084.

[4] A. J. Larkoski, I. Moult and B. Nachman, Jet Substructure at the Large Hadron Collider: A Review of Recent Advances in Theory and Machine Learning (2017), 1709.04464.

[5] D. Guest, K. Cranmer and D. Whiteson, Deep Learning and its Application to LHC Physics, Ann. Rev. Nucl. Part. Sci. 68, 161 (2018), doi:10.1146/annurev-nucl-101917-021019, 1806. 11484 .

[6] K. Albertsson et al., Machine Learning in High Energy Physics Community White Paper, J. Phys. Conf. Ser. 1085(2), 022008 (2018), doi:10.1088/1742-6596/1085/2/022008, 1807. 02876 .

[7] A. Radovic, M. Williams, D. Rousseau, M. Kagan, D. Bonacorsi, A. Himmel, A. Aurisano, K. Terao and T. Wongjirad, Machine learning at the energy and intensity frontiers of particle physics, Nature 560(7716), 41 (2018), doi:10.1038/s41586-018-0361-2.

[8] G. Lemaître, F. Nogueira and C. K. Aridas, Imbalanced-learn: A python toolbox to tackle the curse of imbalanced datasets in machine learning, Journal of Machine Learning Research 18(17), 1 (2017).

[9] R. Aaij et al., Measurement of the time-integrated $C P$ asymmetry in $D^{0} \rightarrow K_{S}^{0} K_{S}^{0}$ decays, JHEP 10, 055 (2015), doi:10.1007/JHEP10(2015)055, 1508.06087. 
[10] M. Britsch, N. Gagunashvili and M. Schmelling, Application of the rule-growing algorithm RIPPER to particle physics analysis, PoS ACAT08, 086 (2008), doi:10.22323/1.070.0086, 0910.1729 .

[11] M. Britsch, N. Gagunashvili and M. Schmelling, Classifying extremely imbalanced data sets, PoS ACAT2010, 047 (2010), doi 10.22323/1.093.0047, 1011.6224.

[12] J. H. Collins, K. Howe and B. Nachman, Anomaly Detection for Resonant New Physics with Machine Learning, Phys. Rev. Lett. 121(24), 241803 (2018), doi:10.1103/PhysRevLett.121.241803, 1805.02664.

[13] M. Farina, Y. Nakai and D. Shih, Searching for New Physics with Deep Autoencoders (2018), 1808.08992 .

[14] T. Heimel, G. Kasieczka, T. Plehn and J. M. Thompson, QCD or What?, SciPost Phys. 6(3), 030 (2019), doi:10.21468/SciPostPhys.6.3.030, 1808.08979.

[15] J. H. Collins, K. Howe and B. Nachman, Extending the search for new resonances with machine learning, Phys. Rev. D99(1), 014038 (2019), doi:10.1103/PhysRevD.99.014038, 1902.02634.

[16] L. M. Dery, B. Nachman, F. Rubbo and A. Schwartzman, Weakly Supervised Classification in High Energy Physics, JHEP 05, 145 (2017), doi:10.1007/JHEP05(2017)145, 1702.00414.

[17] T. Cohen, M. Freytsis and B. Ostdiek, (Machine) Learning to Do More with Less, JHEP 02, 034 (2018), doi:10.1007/JHEP02(2018)034, 1706.09451.

[18] E. M. Metodiev, B. Nachman and J. Thaler, Classification without labels: Learning from mixed samples in high energy physics, JHEP 10, 174 (2017), doi:10.1007/JHEP10(2017)174, 1708.02949 .

[19] https://github.com/christopher-w-murphy/Class-Imbalance-in-WW-Polarization.

[20] Z. Ding, Diversified ensemble classifiers for highly imbalanced data learning and their application in bioinformatics (2011).

[21] M. Kubat, S. Matwin et al., Addressing the curse of imbalanced training sets: one-sided selection, In Icml, vol. 97, pp. 179-186. Nashville, USA (1997).

[22] J. Laurikkala, Improving identification of difficult small classes by balancing class distribution, In S. Quaglini, P. Barahona and S. Andreassen, eds., Artificial Intelligence in Medicine, pp. 63-66. Springer Berlin Heidelberg, Berlin, Heidelberg, ISBN 978-3-540-48229-1 (2001).

[23] I. Mani and I. Zhang, knn approach to unbalanced data distributions: a case study involving information extraction, In Proceedings of workshop on learning from imbalanced datasets, vol. 126 (2003).

[24] B. C. Wallace, K. Small, C. E. Brodley and T. A. Trikalinos, Class imbalance, redux, In Proceedings of the 2011 IEEE 11th International Conference on Data Mining, ICDM '11, pp. 754-763. IEEE Computer Society, Washington, DC, USA, ISBN 978-0-7695-4408-3, doi:10.1109/ICDM.2011.33 (2011). 
[25] M. R. Smith, T. Martinez and C. Giraud-Carrier, An instance level analysis of data complexity, Machine Learning 95(2), 225 (2014), doi:10.1007/s10994-013-5422-z.

[26] H. Han, W.-Y. Wang and B.-H. Mao, Borderline-smote: A new over-sampling method in imbalanced data sets learning, In D.-S. Huang, X.-P. Zhang and G.-B. Huang, eds., Advances in Intelligent Computing, pp. 878-887. Springer Berlin Heidelberg, Berlin, Heidelberg, ISBN 978-3-540-31902-3 (2005).

[27] H. M. Nguyen, E. W. Cooper and K. Kamei, Borderline over\&\#45;sampling for imbalanced data classification, Int. J. Knowl. Eng. Soft Data Paradigm. 3(1), 4 (2011), doi:10.1504/IJKESDP.2011.039875.

[28] G. E. Batista, A. L. Bazzan and M. C. Monard, Balancing training data for automated annotation of keywords: a case study., In WOB, pp. 10-18 (2003).

[29] G. E. A. P. A. Batista, R. C. Prati and M. C. Monard, A study of the behavior of several methods for balancing machine learning training data, SIGKDD Explor. Newsl. 6(1), 20 (2004), doi:10.1145/1007730.1007735.

[30] C. Chen, A. Liaw, L. Breiman et al., Using random forest to learn imbalanced data, University of California, Berkeley 110, 1 (2004).

[31] X. Liu, J. Wu and Z. Zhou, Exploratory undersampling for class-imbalance learning, IEEE Transactions on Systems, Man, and Cybernetics, Part B (Cybernetics) 39(2), 539 (2009), doi: $10.1109 /$ TSMCB.2008.2007853.

[32] C. Seiffert, T. M. Khoshgoftaar, J. Van Hulse and A. Napolitano, Rusboost: A hybrid approach to alleviating class imbalance, IEEE Transactions on Systems, Man, and Cybernetics - Part A: Systems and Humans 40(1), 185 (2010), doi:10.1109/TSMCA.2009.2029559.

[33] S. T. Goh and C. Rudin, Box drawings for learning with imbalanced data, In Proceedings of the 20th ACM SIGKDD International Conference on Knowledge Discovery and Data Mining, KDD '14, pp. 333-342. ACM, New York, NY, USA, ISBN 978-1-4503-2956-9, doi:10.1145/2623330.2623648 (2014).

[34] F. T. Liu, K. M. Ting and Z.-H. Zhou, Isolation-based anomaly detection, ACM Trans. Knowl. Discov. Data 6(1), 3:1 (2012), doi:10.1145/2133360.2133363.

[35] T. R. Bandaragoda, K. M. Ting, D. Albrecht, F. T. Liu and J. R. Wells, Efficient anomaly detection by isolation using nearest neighbour ensemble, In 2014 IEEE International Conference on Data Mining Workshop, pp. 698-705, doi:10.1109/ICDMW.2014.70 (2014).

[36] T.-Y. Lin, P. Goyal, R. Girshick, K. He and P. Dollár, Focal Loss for Dense Object Detection, arXiv e-prints arXiv:1708.02002 (2017), 1708.02002.

[37] K. Feng, H. Hong, K. Tang and J. Wang, Decision making with machine learning and roc curves, arXiv preprint arXiv:1905.02810 (2019).

[38] J. F. Gunion, H. E. Haber and J. Wudka, Sum rules for Higgs bosons, Phys. Rev. D43, 904 (1991), doi:10.1103/PhysRevD.43.904 
[39] B. Grinstein, C. W. Murphy, D. Pirtskhalava and P. Uttayarat, Theoretical Constraints on Additional Higgs Bosons in Light of the 126 GeV Higgs, JHEP 05, 083 (2014), doi:10.1007/JHEP05(2014)083, 1401.0070.

[40] Observation of electroweak production of a same-sign $W$ boson pair in association with two jets in pp collisions at $\sqrt{s}=13$ TeV with the ATLAS detector, Tech. Rep. ATLAS-CONF2018-030, CERN, Geneva (2018).

[41] A. M. Sirunyan et al., Observation of electroweak production of same-sign $W$ boson pairs in the two jet and two same-sign lepton final state in proton-proton collisions at $\sqrt{s}=$ 13 TeV, Phys. Rev. Lett. 120(8), 081801 (2018), doi:10.1103/PhysRevLett.120.081801, 1709.05822.

[42] Prospects for the measurement of the $W^{ \pm} W^{ \pm}$scattering cross section and extraction of the longitudinal scattering component in pp collisions at the High-Luminosity LHC with the ATLAS experiment, Tech. Rep. ATL-PHYS-PUB-2018-052, CERN, Geneva (2018).

[43] Study of $W^{ \pm} W^{ \pm}$production via vector boson scattering at the HL-LHC with the upgraded CMS detector, Tech. Rep. CMS-PAS-FTR-18-005, CERN, Geneva (2018).

[44] P. Azzi et al., Standard Model Physics at the HL-LHC and HE-LHC (2019), 1902.04070.

[45] J. Searcy, L. Huang, M.-A. Pleier and J. Zhu, Determination of the $W W$ polarization fractions in $p p \rightarrow W^{ \pm} W^{ \pm} j j$ using a deep machine learning technique, Phys. Rev. D93(9), 094033 (2016), doi:10.1103/PhysRevD.93.094033, 1510.01691.

[46] J. Lee, N. Chanon, A. Levin, J. Li, M. Lu, Q. Li and Y. Mao, Polarization fraction measurement in same-sign WW scattering using deep learning, Phys. Rev. D99(3), 033004 (2019), doi:10.1103/PhysRevD.99.033004, 1812.07591.

[47] J. Alwall, R. Frederix, S. Frixione, V. Hirschi, F. Maltoni, O. Mattelaer, H. S. Shao, T. Stelzer, P. Torrielli and M. Zaro, The automated computation of tree-level and next-toleading order differential cross sections, and their matching to parton shower simulations, JHEP 07, 079 (2014), doi:10.1007/JHEP07(2014)079, 1405.0301.

[48] F. Pedregosa et al., Scikit-learn: Machine learning in Python, Journal of Machine Learning Research 12, 2825 (2011).

[49] F. Chollet et al., Keras, https://keras.io (2015).

[50] M. Abadi et al., TensorFlow: Large-scale machine learning on heterogeneous systems, Software available from tensorflow.org (2015).

[51] M. Aaboud et al., Search for the Decay of the Higgs Boson to Charm Quarks with the ATLAS Experiment, Phys. Rev. Lett. 120(21), 211802 (2018), doi:10.1103/PhysRevLett.120.211802, 1802.04329.

[52] Search for $H^{0} \rightarrow b \bar{b}$ or $c \bar{c}$ in association with $a W$ or $Z$ boson in the forward region of $p p$ collisions (2016).

[53] J. Ellis, C. W. Murphy, V. Sanz and T. You, Updated Global SMEFT Fit to Higgs, Diboson and Electroweak Data, JHEP 06, 146 (2018), doi:10.1007/JHEP06(2018)146, 1803.03252. 
[54] M. Cepeda et al., Higgs Physics at the HL-LHC and HE-LHC (2019), 1902.00134.

[55] Prospects for $H \rightarrow c \bar{c}$ using Charm Tagging with the ATLAS Experiment at the HL-LHC, Tech. Rep. ATL-PHYS-PUB-2018-016, CERN, Geneva (2018).

[56] J. R. Andersen et al., Handbook of LHC Higgs Cross Sections: 3. Higgs Properties (2013), doi:10.5170/CERN-2013-004, 1307.1347.

[57] M. Aaboud et al., Observation of $H \rightarrow b \bar{b}$ decays and $V H$ production with the ATLAS detector, Phys. Lett. B786, 59 (2018), doi:10.1016/j.physletb.2018.09.013, 1808.08238.

[58] A. M. Sirunyan et al., Observation of Higgs boson decay to bottom quarks, Phys. Rev. Lett. 121(12), 121801 (2018), doi:10.1103/PhysRevLett.121.121801, 1808.08242.

[59] M. Aaboud et al., Searches for exclusive Higgs and $Z$ boson decays into $J / \psi \gamma, \psi(2 S) \gamma$, and $\Upsilon(n S) \gamma$ at $\sqrt{s}=13$ TeV with the ATLAS detector, Phys. Lett. B786, 134 (2018), doi:10.1016/j.physletb.2018.09.024, 1807.00802 .

[60] C. Delaunay, T. Golling, G. Perez and Y. Soreq, Enhanced Higgs boson coupling to charm pairs, Phys. Rev. D89(3), 033014 (2014), doi:10.1103/PhysRevD.89.033014, 1310.7029.

[61] G. Perez, Y. Soreq, E. Stamou and K. Tobioka, Prospects for measuring the Higgs boson coupling to light quarks, Phys. Rev. D93(1), 013001 (2016), doi:10.1103/PhysRevD.93.013001, 1505.06689 .

[62] R. Aaij et al., Identification of beauty and charm quark jets at LHCb, JINST 10(06), P06013 (2015), doi:10.1088/1748-0221/10/06/P06013, 1504.07670.

[63] G. Aad et al., Performance of b-Jet Identification in the ATLAS Experiment, JINST 11(04), P04008 (2016), doi:10.1088/1748-0221/11/04/P04008, 1512.01094.

[64] I. Connelly, Performance and calibration of b-tagging with the ATLAS experiment at LHC Run-2, EPJ Web Conf. 164, 07025 (2017), doi:10.1051/epjconf/201716407025.

[65] A. Lenz, M. Spannowsky and G. Tetlalmatzi-Xolocotzi, Double-charming Higgs boson identification using machine-learning assisted jet shapes, Phys. Rev. D97(1), 016001 (2018), doi:10.1103/PhysRevD.97.016001, 1708.03517.

[66] T. Sjostrand, S. Mrenna and P. Z. Skands, PYTHIA 6.4 Physics and Manual, JHEP 05, 026 (2006), doi:10.1088/1126-6708/2006/05/026, hep-ph/0603175.

[67] M. Cacciari, G. P. Salam and G. Soyez, FastJet User Manual, Eur. Phys. J. C72, 1896 (2012), doi:10.1140/epjc/s10052-012-1896-2, 1111.6097.

[68] M. Cacciari, G. P. Salam and G. Soyez, The anti- $k_{t}$ jet clustering algorithm, JHEP 04, 063 (2008), doi:10.1088/1126-6708/2008/04/063, 0802.1189.

[69] D. Krohn, M. D. Schwartz, T. Lin and W. J. Waalewijn, Jet Charge at the LHC, Phys. Rev. Lett. 110(21), 212001 (2013), doi:10.1103/PhysRevLett.110.212001, 1209.2421.

[70] G. Ke, Q. Meng, T. Finley, T. Wang, W. Chen, W. Ma, Q. Ye and T.-Y. Liu, Lightgbm: A highly efficient gradient boosting decision tree, In I. Guyon, U. V. Luxburg, S. Bengio, H. Wallach, R. Fergus, S. Vishwanathan and R. Garnett, eds., Advances in Neural Information Processing Systems 30, pp. 3146-3154. Curran Associates, Inc. (2017). 
[71] W. J. Waalewijn, Calculating the Charge of a Jet, Phys. Rev. D86, 094030 (2012), doi:10.1103/PhysRevD.86.094030, 1209.3019.

[72] B. Grinstein and C. W. Murphy, Bottom-Quark Forward-Backward Asymmetry in the Standard Model and Beyond, Phys. Rev. Lett. 111, 062003 (2013), doi:10.1103/PhysRevLett.112.239901, 10.1103/PhysRevLett.111.062003, [Erratum: Phys. Rev. Lett.112,no.23,239901(2014)], 1302.6995.

[73] C. W. Murphy, Bottom-Quark Forward-Backward and Charge Asymmetries at Hadron Colliders, Phys. Rev. D92(5), 054003 (2015), doi:10.1103/PhysRevD.92.054003, 1504. 02493

[74] R. Gauld, U. Haisch, B. D. Pecjak and E. Re, Beauty-quark and charm-quark pair production asymmetries at LHCb, Phys. Rev. D92, 034007 (2015), doi:10.1103/PhysRevD.92.034007, 1505.02429 .

[75] R. Gauld, U. Haisch and B. D. Pecjak, Asymmetric heavy-quark hadroproduction at LHCb: Predictions and applications, JHEP 03, 166 (2019), doi:10.1007/JHEP03(2019)166, 1901. 07573 . 